\begin{tabular}{lrr} 
STUDIA ROMANICA & POSNANIENSIA \\
\hline UAM & Vol. 41/1 Poznań 2014
\end{tabular}

MARÍA DEL PILAR ORTIZ LOVILLO

Universidad Veracruzana

\title{
ANÁLISIS MICROGENÉTICO DEL PROCESO DE ENSEÑANZA-APRENDIZAJE DE LA TRADUCCIÓN INFORME DE UNA INVESTIGACIÓN EN PROCESO
}

\begin{abstract}
María del Pilar Ortiz Lovillo, Análisis microgenético del proceso de enseñanza-aprendizaje de la traducción. Informe de una investigación en proceso [Microgenetic analysis of the teaching-learning process of translation Report from an ongoing research], Studia Romanica Posnaniensia, Adam Mickiewicz University Press, Poznań, vol. XLI/1: 2014, pp. 101-118. ISBN 978-83-232-2673-4. ISSN 01372475. eISSN 2084-4158. Doi: 10.7169/strop2014.411.007

The following paper describes the microgenetic analysis of the translation process of Le Petit Chaperon Rouge carried out by six students during the Translation Seminar of Scientific and Literary Texts and its Didactics, instructed at the Institute of Research in Education of the University of Veracruz. This article's purpose is to demonstrate the process which was carried out during the microgenetic analysis, as well as give an initial report of its results and the advantages that this kind of analysis could represent for the translation teaching-learning process. Furthermore, we describe the computer program called El Espia, which enabled us to explore thoroughly and automatically the students' translation process, in order to identify learning scenarios and carried out a microgenetic analysis.
\end{abstract}

Keywords: translation, microgenesis, microgenesis situated, teaching-learning

\section{INTRODUCCIÓN}

La traducción en nuestros días ha llegado a ser indispensable para investigadores, maestros y estudiantes en todos los ámbitos del saber. Es por este motivo que nos parece de la mayor trascendencia la formación de traductores. Con el fin de contribuir a esta tarea, en el Instituto de Investigaciones en Educación impartimos un Seminario de Traducción de Textos Científicos y Literarios del Francés al Español y su Didáctica al que acuden estudiantes de las diferentes licenciaturas de la Universidad Veracruzana.

En este artículo nos propusimos mostrar el trabajo realizado durante el semestre febrero-junio de 2012 con un grupo de seis estudiantes que cursaron el seminario citado. Nos interesaba principalmente saber cuál es el proceso de aprendizaje de la traducción y para lograrlo nos basamos en un análisis microgenético porque permite ver de manera minuciosa lo que ocurre durante el desarrollo de la traducción, como explicaremos más adelante. Por otra parte, nos apoyamos en una herramienta llamada 
El Espía, con la que es posible visualizar segundo a segundo el procedimiento que sigue el estudiante para traducir un texto.

A continuación comentaremos la forma en que se llevó a cabo esta investigación. Si bien es importante mencionar que se trata de un trabajo aún en proceso (y, por lo tanto, no estamos en condiciones de dar resultados definitivos), proporcionamos algunos resultados preliminares, ya que seguimos trabajando en el análisis a profundidad de los datos obtenidos.

Se eligió la traducción del cuento Le Petit Chaperon Rouge de Charles Perrault, por considerar que, a pesar de tratarse de un cuento infantil - aparentemente - sencillo, encierra grandes retos: verbos en desuso, vocabulario para el que es difícil encontrar equivalencias exactas y referencias culturales de antaño.

\section{MICROGÉNESIS}

Algunos autores como Bermejo (2005), Balslev (2006) y Rosenthal (2011) confirman que los orígenes del término «microgénesis» se remontan a Werner (1956), quien afirmaba que «el experimento microgenético ofrece los medios para actualizar o externalizar visiblemente el desarrollo de representaciones internas y los mecanismos que las construyen». Por otro lado, la metodología de Vigotsky (1978) está estrechamente relacionada con las ideas de Werner aunque no haya utilizado el término «microgénesis». Vigotsky señalaba que los procesos de interiorización son creadores de la personalidad y de la conciencia individual y social, además de que son fundamentales para el desarrollo de los procesos psicológicos superiores en los que participan las herramientas de mediación, principalmente el lenguaje.

De igual modo se deben mencionar algunos trabajos de Piaget, que si bien tampoco hace una mención explícita sobre la microgénesis, «sus trabajos sobre la observación y el análisis fino de las conductas motoras en su contexto de realización, por una parte y la perspectiva de comprensión de las transiciones finas de una etapa a otra más compleja del desarrollo, dan la pauta para el análisis microgenético» (Balslev, 2006: 10).

La microgénesis se define como el proceso de adquisición de los conocimientos en un tiempo corto y en una situación particular. Se considera que es posible inferir los procesos de construcción de los conocimientos a partir de los indicios extraídos de observaciones.

Así, el método microgenético es el único que es capaz de centrarse a la vez en los procesos finos de desarrollo cognitivo y en los contextos específicos donde se desarrollan esos procesos. «Un análisis microgenético implica una observación microscópica de una situación didáctica, implica también la idea de génesis, es decir una evolución al interior de determinada secuencia» (Saada-Robert y Balslev, 2006: 1). 
En resumen, el método microgenético consiste en estudiar de manera individual a un grupo de sujetos durante una serie de sesiones, en las cuales se le presenta a cada uno una tarea cuya solución implica la aplicación de estrategias de aprendizaje.

\subsection{LAS MICROGÉNESIS SITUADAS}

Balslev (2006: 69) señala que las microgénesis situadas se diferencian de las otras corrientes microgenéticas por la importancia que se le da al carácter situado de esos procesos, en oposición a los procesos estudiados en laboratorio.

El estudio de las microgénesis situadas se interesa principalmente en las significaciones, es decir, en los conocimientos locales y contextuales atribuidos y construidos por los participantes de la interacción.

El estudio de las microgénesis situadas, de acuerdo con Saada-Robert y Balslev (2006: 86), abreva en la psicología del desarrollo constructivista, principalmente en la cuestión de la transformación de los conocimientos y, por otra parte, en el análisis de los procesos socio-cognitivos que permiten explicar esa transformación, al menos en el momento situado de los aprendizajes. También tiene que ver con el estudio de las microinteracciones, en particular cuando esas interacciones toman su lugar en tiempo real en las situaciones de enseñanza-aprendizaje.

\subsection{LAS MICROGÉNESIS DIDÁCTICAS}

Por su parte, las microgénesis didácticas se insertan en el cruce de los campos de las microgénesis situadas y la didáctica, cuando ésta se interesa en la última fase de la transposición de los saberes en conocimientos en el estudiante, la fase de la enseñanza efectiva, así como en el sistema tríadico saber-enseñanza-aprendizaje (Saada-Robert y Balslev, 2006).

Las microgénesis didácticas son estudiadas principalmente en un contexto escolar y tienen que ver con aprendizajes instituidos y en un contexto educativo para los aprendizajes llamados informales o incluso en contexto de formación de adultos (Balslev, 2004).

El estudio de las microgénesis didácticas es un tipo de estudio de microgénesis situadas que tiene la particularidad de referirse a situaciones de aprendizaje formales, mientras que el estudio de las microgénesis situadas puede igualmente referirse a situaciones de aprendizaje informales, por ejemplo, cuando se estudia a un niño que aprende cómo remontar un juguete mecánico con su madre (Saada-Robert y Balslev, 2004). 


\subsection{VENTAJAS Y APLICACIONES DEL ANÁLISIS MICROGENÉTICO}

Son varias las ventajas que ofrece el análisis microgenético: el cambio puede observarse directamente mientras está ocurriendo y permite estudiar varios aspectos del cambio, como la secuencia de los comportamientos, la rapidez de su ocurrencia, el grado de generalización, las diferencias individuales y sus causas (Siegler, y Crowley, 1992).

El análisis microgenético se ha aplicado con resultados satisfactorios en diferentes campos: en el ámbito del descubrimiento e invención, la aritmética, el razonamiento científico, la memoria, la lectura y en las dificultades de aprendizaje, aunque hasta el momento no se ha localizado ningún estudio sobre la microgénesis destinada al aprendizaje de la traducción.

Para el presente estudio hemos utilizado el análisis microgenético para examinar de manera microscópica el proceso de aprendizaje de los estudiantes de traducción que cursaron el Seminario de Traducción de Textos Científicos y Literarios del Francés al Español y su Didáctica. Durante las sesiones se fueron observando los cambios en el aprendizaje de la traducción del texto hasta que se consideró que cumplía con las características esperadas. Se grabaron las sesiones en video y audio a fin de que pudiera analizarse la conducta cognitiva. En conjunción con esto, y con el fin de observar con mayor minuciosidad el proceso de aprendizaje de los estudiantes de traducción, utilizamos el programa informático llamado El Espía.

\subsubsection{EL ESPÍA}

El Espía es un programa informático recientemente diseñado en el Instituto de Investigaciones en Educación de la Universidad Veracruzana y pretende ser empleado para la exploración de los procesos psicolingüísticos de producción de textos mediante el registro de la posición y el tiempo asociado a cada carácter introducido a través del teclado. El sistema genera un reporte que permite la reconstrucción del proceso de producción y sus operaciones básicas como agregar, borrar, sustituir y desplazar caracteres dentro del texto y la detección de pausas en el proceso de escritura (Ramírez y Vaca, 2011).

Uno de los propósitos es ilustrar con todo detalle cómo realizan los estudiantes sus traducciones en computadora a fin de que, mediante un análisis a profundidad del corpus traducido basado en aspectos lingüísticos, se pueda realizar un análisis microgenético entendido como un medio para analizar y observar directamente el cambio, la transición y sus mecanismos evolutivos.

Así, fue posible observar con todo detalle los trabajos de los estudiantes y darnos cuenta de cómo puede auxiliarnos al momento de evaluar sus traducciones, ya que nos permitió ver con mayor objetividad sus dudas, aciertos y errores. 
Con los datos reportados por el programa citado fue posible generar una visualización de cómo el estudiante fue traduciendo y elaborando el texto, y pudimos contar así con un reporte detallado de toda la actividad para conocer su progreso en el aprendizaje de la traducción.

\subsubsection{EL PROCESO DE INVESTIGACIÓN}

El objeto de estudio fueron las situaciones de aprendizaje de la traducción del francés al español. El escenario, el Seminario de Traducción de Textos Científicos y Literarios del Francés al Español y su Didáctica. Los informantes fueron seis, dos hombres y cuatro mujeres, y para identificarlos utilizamos los seudónimos siguientes: Daniel, Aris, Leonor, Nicki, Aline e Isis. Sus edades fluctúan entre 20 y 33 años, todos son de nacionalidad mexicana y su lengua materna es el español. Cinco de los estudiantes cursaban en ese momento el sexto semestre de la Licenciatura en Lengua Francesa, mientras que otro cursaba la Licenciatura en Derecho.

Se trabajó durante dieciséis sesiones, con una duración de dos horas cada una. Al inicio se diseñó una prueba que consistió en traducir del francés al español el primer párrafo del cuento Le Petit Chaperon Rouge de Charles Perrault. Durante el proceso de realización de la tarea, la profesora fue observando el proceso de traducción y registrando los problemas que encontraban los estudiantes, a fin de obtener una muestra inicial de su competencia traductora.

Antes de realizar la segunda versión de la traducción del primer párrafo, la profesora explicó cómo resolver algunos de los problemas encontrados en el texto. Los estudiantes hicieron un trabajo de reflexión en pareja, el cual fue grabado para poder registrar sus comentarios, dudas e inquietudes y conocer un poco más la manera en la que iban analizando la tarea y construyendo su aprendizaje.

Para realizar la tercera versión se les solicitó que realizaran una nueva traducción individual del mismo párrafo para ver sus avances o retrocesos y analizar los problemas a los que se enfrentaban en dicha traducción, para ilustrarlo mejor utilizamos el programa El Espía.

Más adelante se trabajó con otros textos literarios para obtener nuevas muestras de los avances en el aprendizaje de la traducción y para evitar que les resultara tedioso trabajar varias sesiones con un solo texto.

Algunos estudiantes tuvieron que hacer hasta cinco versiones para considerar que su traducción cumplía con las condiciones necesarias, es decir, que la traducción se podía leer como un original en español, presentaba una trasmisión fiel de la información y el sentido del texto, además de que ya no aparecían errores de semántica, sintaxis, estilo, lexicología ni ortografía.

Se filmaron algunas sesiones donde la profesora daba explicaciones sobre las dificultades encontradas en el cuento o aclaraba dudas sobre las dificultades de la traducción, con el fin de determinar la importancia de la interacción. 
En esta investigación nos basamos en el estudio de caso, la observación participante, el uso del video en la observación, el uso de datos históricos y biográficos y el análisis microgenético.

Nos centraremos aquí en el análisis microgenético porque permitió seguir un procedimiento de construcción progresiva ya que en cada etapa fuimos observando el antes y el después para poder determinar si hubo algún cambio en el proceso de aprendizaje. Se utilizaron varias fases a fin de obtener los datos que se iban a analizar. Cada una de ellas corresponde a un procedimiento específico.

En la primera fase recogimos los datos brutos, es decir, sin procesar, como: las notas de observación de las sesiones del Seminario de Traducción, las traducciones de los estudiantes del cuento y, posteriormente, las traducciones del mismo texto utilizando el programa El Espía.

En la segunda fase, de acuerdo con los errores que se presentaron con mayor frecuencia en las traducciones, se determinaron las categorías: semántica, sintaxis, lexicología, ortografía, contexto cultural, cohesión verbal, omisión y agregado.

En la tercera fase se hizo el recorte del texto en unidades de traducción, tanto del original en francés como de las traducciones de los seis estudiantes, para establecer comparaciones posteriormente.

En la cuarta fase se elaboraron cuadros con diferentes columnas, en la primera aparece el texto en francés y en las siguientes los diferentes párrafos que los estudiantes fueron produciendo y se resaltaron los errores de acuerdo a las categorías mencionadas. Además, se analizó cada párrafo y se compararon las diferentes versiones de cada estudiante para observar su avance

En la quinta fase se determinó la importancia de la interacción profesor-estudiante y estudiante-estudiante en el proceso de aprendizaje de la traducción.

\subsubsection{ANÁLISIS DE RESULTADOS}

Como mencionamos anteriormente, para poder realizar el análisis utilizamos un procedimiento que constituyó el fundamento del microanálisis, el cual estuvo constituido por dos operaciones: la elaboración de categorías y la confección de cuadros donde se insertaron todas las unidades de traducción, párrafo por párrafo.

Los criterios para definir las categorías fueron: a) la pertinencia de las categorías en relación con la problemática teórica, y b) que ayudaran a responder la pregunta de investigación: ¿Cómo se lleva a cabo el proceso de aprendizaje de la traducción de francés al español desde el análisis microgenético?, y las preguntas específicas: ¿Cuáles son los componentes del saber, observables e identificables en las traducciones de los alumnos a través del análisis textual? ¿Cuáles son los nexos que existen entre profesor y estudiante y la construcción del aprendizaje? Y ¿cómo se pueden analizar los cambios evolutivos en el proceso de aprendizaje de la traducción? 
Del mismo modo, para considerar que las categorías eran pertinentes, tenían que contar con tres elementos: ser necesarias, estar ligadas entre sí de manera coherente y que aparecieran con frecuencia en los párrafos analizados y en las unidades de traducción. También se cuidó que tuvieran relación con la problemática teórica que nos sirvió de marco y nos permitiera responder a las preguntas de investigación.

En nuestra investigación la unidad de análisis fue la unidad de traducción (UT). Esta última de acuerdo con Christiane Nord, (1998) se ha definido como «la unidad de la lengua o el texto de partida tratada por el traductor en el proceso de traducción»; ella comenta que hasta el momento los estudiosos de la traducción no se han puesto de acuerdo en lo referente a los proporciones ideales de una UT ni en el nivel o rango lingüístico en el que se localiza, ni tampoco si debería constituirse por morfemas, palabras, frases, oraciones o incluso textos completos.

Por lo tanto, la división en unidades de traducción se hizo de manera arbitraria. Nos planteamos dos preguntas en relación con esta operación: ¿Cuál es el tamaño que debían tener? Y ¿cómo interpretar un enunciado dividido en unidades de traducción tal como los estudiantes las tradujeron y las comprendieron? Para empezar, consideramos que las unidades de traducción debían corresponder a palabras clave, expresiones clave y proposiciones de párrafos que contuvieran un único y mismo sentido, pues en las microgénesis situadas se trata de que el investigador reconstituya el sentido oculto en los enunciados incorrectos.

A continuación presentamos un cuadro que contiene el primer párrafo de Le Petit Chaperon Rouge en francés, así como la primera y última versión de la traducción de los estudiantes. Se resaltaron errores específicos en relación con las categorías de cohesión verbal, lexicología y semántica. Estos representan simplemente los errores más significativos de este primer párrafo y componen nuestro primer acercamiento al análisis microgenético del proceso de traducción de los estudiantes.

Comparación de las traducciones de los estudiantes

Le Petit Chaperon Rouge

\begin{tabular}{|l|l|l|l|}
\hline \multirow{7}{*}{ Daniel } & $\begin{array}{l}\text { Versión en francés } \\
\text { 1. Le Petit Chaperon } \\
\text { rouge. } \\
\text { Charles Perrault }\end{array}$ & 1. Sin titulo ni autor & $\begin{array}{c}\text { Última versión } \\
\text { 1. Caperucita Roja. } \\
\text { Charles Perrault }\end{array}$ \\
\cline { 2 - 4 } & 2. Il était une fois & 2. Había una vez & 2. Erase una vez \\
\cline { 2 - 4 } & $\begin{array}{l}\text { 3. une petite fille de } \\
\text { Village, }\end{array}$ & $\begin{array}{l}\text { 3. una pequeña niña de } \\
\text { pueblo, }\end{array}$ & $\begin{array}{l}\text { 3. una niña que vivía en } \\
\text { una aldea, }\end{array}$ \\
\cline { 2 - 4 } & $\begin{array}{l}\text { 4. la plus jolie qu'on eût } \\
\text { su voir; }\end{array}$ & $\begin{array}{c}\text { 4. la más bella que se } \\
\text { haya visto, }\end{array}$ & $\begin{array}{c}\text { 4. la niña más bella que } \\
\text { se hubiera visto. }\end{array}$ \\
\hline
\end{tabular}




\begin{tabular}{|c|c|c|c|}
\hline & $\begin{array}{l}\text { 5. sa mère en était folle, } \\
\text { et sa mère- grand } \\
\text { plus folle encore. }\end{array}$ & $\begin{array}{l}\text { 5. su madre estaba } \\
\text { enferma y su abuela } \\
\text { aún más. }\end{array}$ & $\begin{array}{l}\text { 5. Su mamá estaba } \\
\text { loca por su hija y su } \\
\text { abuelita aún más }\end{array}$ \\
\hline & $\begin{array}{l}\text { 6. Cette bonne femme } \\
\text { lui fit faire un petit } \\
\text { chaperon rouge. }\end{array}$ & $\begin{array}{l}\text { 6. Esta buena mujer le } \\
\text { tejió una pequeña } \\
\text { caperucita roja, }\end{array}$ & $\begin{array}{l}\text { 6. Esta buena mujer le } \\
\text { tejió a la pequeña una } \\
\text { caperuza roja }\end{array}$ \\
\hline & 7. qui lui seyait si bien, & $\begin{array}{l}\text { 7. que le quedaba tan } \\
\text { bien, }\end{array}$ & $\begin{array}{l}\text { 7. que le quedaba tan } \\
\text { bien, }\end{array}$ \\
\hline & 8. que partout & 8. que todos en el pueblo & 8. que por todos lados \\
\hline & $\begin{array}{l}\text { 9. On l'appelait le Petit } \\
\text { Chaperon rouge. }\end{array}$ & $\begin{array}{l}\text { 9. la llamaban la pequeña } \\
\text { Caperuza Roja. }\end{array}$ & $\begin{array}{l}\text { 9. le llamaban: } \\
\text { Caperucita Roja. }\end{array}$ \\
\hline & $\begin{array}{l}\text { 10. Un jour sa mère } \\
\text { ayant fait et cuit des } \\
\text { galettes, lui dit: }\end{array}$ & $\begin{array}{l}\text { 10. Un día su madre } \\
\text { habiendo cocinado } \\
\text { panquecillos le dijo: }\end{array}$ & $\begin{array}{l}\text { 10. Un día, su mamá, } \\
\text { que había hecho } \\
\text { pastelillos, le dijo: }\end{array}$ \\
\hline & $\begin{array}{l}\text { 11. Va voir comme se } \\
\text { porte ta mère-grand, }\end{array}$ & $\begin{array}{l}\text { 11. "Ve a ver cómo se } \\
\text { encuentra tu abuela, }\end{array}$ & $\begin{array}{l}\text { 11. ve a ver cómo está tu } \\
\text { abuelita, }\end{array}$ \\
\hline & $\begin{array}{l}\text { 12. Car on m'as dit qu'elle } \\
\text { était malade, }\end{array}$ & $\begin{array}{l}\text { 12. ya que me han dicho } \\
\text { que se encuentra } \\
\text { enferma, }\end{array}$ & $\begin{array}{l}\text { 12. pues me han dicho que } \\
\text { estaba enferma. }\end{array}$ \\
\hline & $\begin{array}{l}\text { 13. Porte lui une galette et } \\
\text { ce petit pot de beurre. }\end{array}$ & $\begin{array}{l}\text { 13. llévale un panquecillo } \\
\text { y un bote de } \\
\text { mermelada }\end{array}$ & $\begin{array}{l}\text { 13. Llévale este pastelillo } \\
\text { y este tarro de } \\
\text { mantequilla. }\end{array}$ \\
\hline & $\begin{array}{l}\text { 14. Le Petit Chaperon } \\
\text { rouge partit aussitôt } \\
\text { pour aller chez sa } \\
\text { mère-grand, }\end{array}$ & $\begin{array}{l}\text { 14. La pequeña } \\
\text { Caperuza Roja partio } \\
\text { rápidamente para ver } \\
\text { a su abuela, }\end{array}$ & $\begin{array}{l}\text { 14. Caperucita Roja salió } \\
\text { rápidamente de su } \\
\text { casa para ir a la casa } \\
\text { de su abuela, }\end{array}$ \\
\hline & $\begin{array}{l}\text { 15. qui demeurait dans un } \\
\text { autre village. }\end{array}$ & $\begin{array}{l}\text { 15. quien habitaba en otro } \\
\text { poblado. }\end{array}$ & $\begin{array}{l}\text { 15. quien vivía en otra } \\
\text { aldea. }\end{array}$ \\
\hline Nicki & $\begin{array}{l}\text { 1. Le Petit Chaperon } \\
\text { rouge. } \\
\text { Charles Perrault }\end{array}$ & 1. Sin título ni autor & $\begin{array}{l}\text { 1. Caperucita Roja. } \\
\text { Charles Perrault }\end{array}$ \\
\hline & 2. Il était une fois & 2. Érase una vez, & 2. Érase una vez, \\
\hline & $\begin{array}{l}\text { 3. une petite fille de } \\
\text { Village, }\end{array}$ & 3. una niñita de pueblo, & 3. una niñita de campo, \\
\hline & $\begin{array}{l}\text { 4. la plus jolie qu'on ê̂t } \\
\text { su voir; }\end{array}$ & $\begin{array}{l}\text { 4. la más bonita que se } \\
\text { haya visto, }\end{array}$ & $\begin{array}{l}\text { 4. la más bonita que se } \\
\text { haya visto; }\end{array}$ \\
\hline & $\begin{array}{l}\text { 5. sa mère en était folle, } \\
\text { et sa mère-grand plus } \\
\text { folle encore. }\end{array}$ & $\begin{array}{l}\text { 5. su madre estaba loca } \\
\text { y su abuela lo estaba } \\
\text { aún más. }\end{array}$ & $\begin{array}{l}\text { 5. su mamá estaba loca } \\
\text { por ella y su abuelita } \\
\text { aún más. }\end{array}$ \\
\hline & $\begin{array}{l}\text { 6. Cette bonne femme } \\
\text { lui fit faire un petit } \\
\text { chaperon rouge, }\end{array}$ & $\begin{array}{l}\text { 6. Esa buena mujer le } \\
\text { hizo una caperuza roja }\end{array}$ & $\begin{array}{l}\text { 6. Esta buena mujer le } \\
\text { mandó a hacer una } \\
\text { caperuza roja }\end{array}$ \\
\hline
\end{tabular}




\begin{tabular}{|c|c|c|c|}
\hline & 7. qui lui seyait si bien, & 7. y le quedaba $\tan$ bien & 7. que le sentaba tan bien \\
\hline & 8. que partout & 8. que todo el mundo & 8. que por doquier \\
\hline & $\begin{array}{l}\text { 9. on l'appelait le Petit } \\
\text { Chaperon rouge }\end{array}$ & $\begin{array}{l}\text { 9. la conocía como } \\
\text { Caperucita Roja. }\end{array}$ & $\begin{array}{l}\text { 9. la llamaban Caperucita } \\
\text { Roja. }\end{array}$ \\
\hline & $\begin{array}{l}\text { 10. Un jour sa mère } \\
\text { ayant fait et cuit des } \\
\text { galettes, lui dit: }\end{array}$ & $\begin{array}{l}\text { 10. Un día su mamá hizo } \\
\text { galletas y le dijo: }\end{array}$ & $\begin{array}{l}\text { 10. Un día, su mamá hizo } \\
\text { pastelillos y le dijo: }\end{array}$ \\
\hline & $\begin{array}{l}\text { 11. Va voir comme se } \\
\text { porte ta mère-grand, }\end{array}$ & $\begin{array}{l}\text { 11. Ve a ver cómo está tu } \\
\text { abuela, }\end{array}$ & $\begin{array}{l}\text { 11. Ve a ver cómo se } \\
\text { encuentra tu abuelita, }\end{array}$ \\
\hline & $\begin{array}{l}\text { 12. car on m'as dit qu'elle } \\
\text { était malade, }\end{array}$ & $\begin{array}{l}\text { 12. ya que me dijeron que } \\
\text { está enferma, }\end{array}$ & $\begin{array}{l}\text { 12. pues me dijeron que } \\
\text { estaba enferma. }\end{array}$ \\
\hline & $\begin{array}{l}\text { 13. porte lui une galette et } \\
\text { ce petit pot de beurre. }\end{array}$ & $\begin{array}{l}\text { 13. llévale unas galletas y } \\
\text { un tarro de mermelada }\end{array}$ & $\begin{array}{l}\text { 13. Llévale un pastelillo } \\
\text { y un tarro de } \\
\text { mantequilla. }\end{array}$ \\
\hline & $\begin{array}{l}\text { 14. Le Petit Chaperon } \\
\text { rouge partit aussitôt } \\
\text { pour aller chez sa } \\
\text { mère-grand, }\end{array}$ & $\begin{array}{l}\text { 14. Caperucita roja partió } \\
\text { enseguida hacia la casa } \\
\text { de su abuela }\end{array}$ & $\begin{array}{l}\text { 14. Caperucita Roja partió } \\
\text { enseguida hacia casa } \\
\text { de su abuelita, }\end{array}$ \\
\hline & $\begin{array}{l}\text { 15. qui demeurait dans un } \\
\text { autre village. }\end{array}$ & $\begin{array}{l}\text { 15. que se encontraba en } \\
\text { otro pueblo. }\end{array}$ & $\begin{array}{l}\text { 15. que vivía en otro } \\
\text { pueblo. }\end{array}$ \\
\hline \multirow[t]{10}{*}{ Leonor } & $\begin{array}{l}\text { 1. Le Petit Chaperon } \\
\text { rouge. } \\
\text { Charles Perrault }\end{array}$ & $\begin{array}{l}\text { 1. La caperucita roja. Sin } \\
\text { autor }\end{array}$ & $\begin{array}{l}\text { 1. La Caperucita Roja. } \\
\text { Charles Perrault }\end{array}$ \\
\hline & 2. Il était une fois & 2. Había una vez & 2. Había una vez \\
\hline & $\begin{array}{l}\text { 3. une petite fille de } \\
\text { Village, }\end{array}$ & $\begin{array}{l}\text { 3. una pequeña niña de } \\
\text { pueblo, }\end{array}$ & $\begin{array}{l}\text { 3. una pequeña } \\
\text { pueblerina, }\end{array}$ \\
\hline & $\begin{array}{l}\text { 4. la plus jolie qu'on eût } \\
\text { su voir; }\end{array}$ & $\begin{array}{l}\text { 4. la más feliz que se } \\
\text { hubiera visto; }\end{array}$ & $\begin{array}{l}\text { 4. la más bonita que se } \\
\text { hubiera podido ver. }\end{array}$ \\
\hline & $\begin{array}{l}\text { 5. sa mère en était folle, } \\
\text { et sa mère-grand plus } \\
\text { folle encore. }\end{array}$ & $\begin{array}{l}\text { 5. su madre estaba loca } \\
\text { y su abuela todavía } \\
\text { más loca. }\end{array}$ & $\begin{array}{l}\text { 5. Su mamá estaba loca } \\
\text { por ella y su abuelita } \\
\text { aún más. }\end{array}$ \\
\hline & $\begin{array}{l}\text { 6. Cette bonne femme } \\
\text { lui fit faire un petit } \\
\text { chaperon rouge, }\end{array}$ & $\begin{array}{l}\text { 6. Esta buena mujer le } \\
\text { mandó hacer una } \\
\text { pequeña capa roja, }\end{array}$ & $\begin{array}{l}\text { 6. Esta buena mujer le } \\
\text { mandó a hacer una } \\
\text { caperucita roja, }\end{array}$ \\
\hline & 7. qui lui seyait si bien, & 7. que le quedaba tan bien, & 7. que le quedaba tan bien, \\
\hline & 8. que partout & 8. que por doquier & 8. que por doquier \\
\hline & $\begin{array}{l}\text { 9. on l'appelait le Petit } \\
\text { Chaperon rouge, }\end{array}$ & $\begin{array}{l}\text { 9. la llamaban Caperucita } \\
\text { roja. }\end{array}$ & $\begin{array}{l}\text { 9. la llamaban Caperucita } \\
\text { Roja. }\end{array}$ \\
\hline & $\begin{array}{l}\text { 10. Un jour sa mère } \\
\text { ayant fait et cuit des } \\
\text { galettes, lui dit: }\end{array}$ & $\begin{array}{l}\text { 10. Un día su madre } \\
\text { habiendo cocido y } \\
\text { hecho tartas, le dijo: }\end{array}$ & $\begin{array}{l}\text { 10. Un día, su madre, } \\
\text { habiendo cocinado } \\
\text { pastelillos, le dijo: }\end{array}$ \\
\hline
\end{tabular}




\begin{tabular}{|c|c|c|c|}
\hline & $\begin{array}{l}\text { 11. Va voir comme se } \\
\text { porte ta mère-grand, }\end{array}$ & $\begin{array}{l}\text { 11. ve a ver cómo se } \\
\text { encuentra tu abuela, }\end{array}$ & $\begin{array}{l}\text { 11. ve a ver cómo se } \\
\text { encuentra tu abuelita, }\end{array}$ \\
\hline & $\begin{array}{l}\text { 12. car on m'as dit qu'elle } \\
\text { était malade, }\end{array}$ & $\begin{array}{l}\text { 12. ya que me dijeron que } \\
\text { estaba enferma, }\end{array}$ & $\begin{array}{l}\text { 12. ya que me dijeron que } \\
\text { estaba enferma. }\end{array}$ \\
\hline & $\begin{array}{l}\text { 13. porte-lui une galette et } \\
\text { ce petit pot de beurre. }\end{array}$ & $\begin{array}{l}\text { 13. llévale una tarta y } \\
\text { este frasquito de } \\
\text { mantequilla. }\end{array}$ & $\begin{array}{l}\text { 13. Llévale un pastelillo } \\
\text { y este tarrito de } \\
\text { mantequilla. }\end{array}$ \\
\hline & $\begin{array}{l}\text { 14. Le Petit Chaperon } \\
\text { rouge partit aussitôt } \\
\text { pour aller chez sa } \\
\text { mère-grand, }\end{array}$ & $\begin{array}{l}\text { 14. Caperucita partió } \\
\text { pronto para ir a casa de } \\
\text { su abuela, }\end{array}$ & $\begin{array}{l}\text { 14. La Caperucita Roja } \\
\text { salió rápidamente } \\
\text { hacia la casa de su } \\
\text { abuelita, }\end{array}$ \\
\hline & $\begin{array}{l}\text { 15. qui demeurait dans un } \\
\text { autre village. }\end{array}$ & $\begin{array}{l}\text { 15. que vivía en otro } \\
\text { pueblo }\end{array}$ & $\begin{array}{l}\text { 15. que vivía en otro } \\
\text { pueblo. }\end{array}$ \\
\hline \multirow[t]{13}{*}{ Aline } & $\begin{array}{l}\text { 1. Le Petit Chaperon } \\
\text { rouge. } \\
\text { Charles Perrault }\end{array}$ & 1. Sin título ni autor & $\begin{array}{l}\text { 1. LE PETITE } \\
\text { CHAPERON ROUGE } \\
\text { Charles Perrault }\end{array}$ \\
\hline & 2. Il était une fois & 2. Había una vez & 2. Había una vez \\
\hline & $\begin{array}{l}\text { 3. une petite fille de } \\
\text { Village, }\end{array}$ & $\begin{array}{l}\text { 3. una pequeña } \\
\text { pueblerina, }\end{array}$ & $\begin{array}{l}\text { 3. una pequeña } \\
\text { pueblerina, }\end{array}$ \\
\hline & $\begin{array}{l}\text { 4. la plus jolie qu'on ê̂t } \\
\text { su voir; }\end{array}$ & $\begin{array}{l}\text { 4. la más bonita que se } \\
\text { haya visto, }\end{array}$ & $\begin{array}{l}\text { 4. la más bonita que se } \\
\text { haya visto, }\end{array}$ \\
\hline & $\begin{array}{l}\text { 5. sa mère en était folle, } \\
\text { et sa mère-grand plus } \\
\text { folle encore. }\end{array}$ & $\begin{array}{l}\text { 5. su madre estaba loca } \\
\text { por ella y su abuela } \\
\text { todavía más. }\end{array}$ & $\begin{array}{l}\text { 5. su madre estaba loca } \\
\text { por ella y su abuela } \\
\text { todavía más. }\end{array}$ \\
\hline & $\begin{array}{l}\text { 6. Cette bonne femme } \\
\text { lui fit faire un petit } \\
\text { chaperon rouge. }\end{array}$ & $\begin{array}{l}\text { 6. Esta buena mujer le } \\
\text { mandó a hacer una } \\
\text { caperucita roja }\end{array}$ & $\begin{array}{l}\text { 6. Esta buena mujer le } \\
\text { mandó hacer una } \\
\text { caperucita roja. }\end{array}$ \\
\hline & 7. qui lui seyait si bien, & $\begin{array}{l}\text { 7. que le quedaba muy } \\
\text { bien }\end{array}$ & $\begin{array}{l}\text { 7. que le quedaba muy } \\
\text { bien }\end{array}$ \\
\hline & 8. que partout & 8. y por todos lados & 8. y por todos lados \\
\hline & $\begin{array}{l}\text { 9. on l'appelait le Petit } \\
\text { Chaperon rouge. }\end{array}$ & $\begin{array}{l}\text { 9. le llamaban caperucita } \\
\text { roja. }\end{array}$ & $\begin{array}{l}\text { 9. le llamaban caperucita } \\
\text { roja. }\end{array}$ \\
\hline & $\begin{array}{l}\text { 10. Un jour sa mère } \\
\text { ayant fait et cuit des } \\
\text { galettes, lui dit: }\end{array}$ & $\begin{array}{l}\text { 10. Un día su madre } \\
\text { había } \\
\text { le dijo }\end{array}$ & $\begin{array}{l}\text { 10. Un día su madre } \\
\text { había horneado unos } \\
\text { pastelillos y le dijo: }\end{array}$ \\
\hline & $\begin{array}{l}\text { 11. Va voir comme se } \\
\text { porte ta mère-grand, }\end{array}$ & $\begin{array}{l}\text { 11. Ve a ver como esta tu } \\
\text { abuela }\end{array}$ & $\begin{array}{l}\text { 11. Ve a ver como está tu } \\
\text { abuelita, }\end{array}$ \\
\hline & $\begin{array}{l}\text { 12. Car on m'as dit qu'elle } \\
\text { était malade, }\end{array}$ & $\begin{array}{l}\text { 12. porque me han dicho } \\
\text { que estaba enferma, }\end{array}$ & $\begin{array}{l}\text { 12. porque me han dicho } \\
\text { que estaba enferma, }\end{array}$ \\
\hline & $\begin{array}{l}\text { 13. Porte lui une galette et } \\
\text { ce petit pot de beurre. }\end{array}$ & $\begin{array}{l}\text { 13. llevale una } \\
\text { este tarrito de } \\
\text { mantequilla. }\end{array}$ & $\begin{array}{l}\text { 13. llévale un pastelito } \\
\text { y este tarrito de } \\
\text { mantequilla. }\end{array}$ \\
\hline
\end{tabular}




\begin{tabular}{|c|c|c|c|}
\hline & $\begin{array}{l}\text { 14. Le Petit Chaperon } \\
\text { rouge partit aussitôt } \\
\text { pour aller chez sa } \\
\text { mère-grand, }\end{array}$ & $\begin{array}{l}\text { 14. Caperucita Roja salió } \\
\text { muy temprano hacia } \\
\text { casa de su abuela }\end{array}$ & $\begin{array}{l}\text { 14. Caperucita Roja salió } \\
\text { enseguida hacia casa } \\
\text { de su abuelita }\end{array}$ \\
\hline & $\begin{array}{l}\text { 15. qui demeurait dans un } \\
\text { autre village. }\end{array}$ & $\begin{array}{l}\text { 15. que vivía en otro } \\
\text { pueblo. }\end{array}$ & $\begin{array}{l}\text { 15. que vivía en otro } \\
\text { pueblo. }\end{array}$ \\
\hline \multirow[t]{13}{*}{ Aris } & $\begin{array}{l}\text { 1. Le Petit Chaperon } \\
\text { rouge. } \\
\text { Charles Perrault }\end{array}$ & $\begin{array}{l}\text { 1. Caperucita Roja. } \\
\text { Charles Perrault }\end{array}$ & 1. Sin título ni autor \\
\hline & 2. Il était une fois & 2. Erase una vez, & 2. Había una vez, \\
\hline & $\begin{array}{l}\text { 3. une petite fille de } \\
\text { Village, }\end{array}$ & 3. una aldeanita, & 3. una aldeanita, \\
\hline & $\begin{array}{l}\text { 4. la plus jolie qu'on eût } \\
\text { su voir; }\end{array}$ & $\begin{array}{l}\text { 4. la más linda de la cual } \\
\text { se haya tenido noticia, }\end{array}$ & $\begin{array}{l}\text { 4. la más linda que se } \\
\text { haya visto. }\end{array}$ \\
\hline & $\begin{array}{l}\text { 5. sa mère en était folle, } \\
\text { et sa mère-grand plus } \\
\text { folle encore. }\end{array}$ & $\begin{array}{l}\text { 5. su madre estaba loca } \\
\text { por ella* y su abuela } \\
\text { aún más. *A quien } \\
\text { su madre amaba con } \\
\text { locura y su abuela, } \\
\text { aún más. }\end{array}$ & $\begin{array}{l}\text { 5. Su mamá la amaba } \\
\text { con locura y su } \\
\text { abuelita aún más. }\end{array}$ \\
\hline & $\begin{array}{l}\text { 6. Cette bonne femme } \\
\text { lui fit faire un petit } \\
\text { chaperon rouge, }\end{array}$ & $\begin{array}{l}\text { 6. Esta última, le hizo } \\
\text { confeccionar una } \\
\text { caperucita roja }\end{array}$ & $\begin{array}{l}\text { 6. Esta buena mujer le } \\
\text { hizo confeccionar una } \\
\text { caperuza roja }\end{array}$ \\
\hline & 7. qui lui seyait si bien, & $\begin{array}{l}\text { 7. que le sentaba tan } \\
\text { bien, }\end{array}$ & $\begin{array}{l}\text { 7. que le sentaba tan } \\
\text { bien, }\end{array}$ \\
\hline & 8. que partout & 8. que por doquiera & 8. que por doquier \\
\hline & $\begin{array}{l}\text { 9. on l'appelait le Petit } \\
\text { Chaperon rouge, }\end{array}$ & $\begin{array}{l}\text { 9. le llamaban, } \\
\text { Caperucita roja }\end{array}$ & $\begin{array}{l}\text { 9. le llamaban: La } \\
\text { Caperucita Roja. }\end{array}$ \\
\hline & $\begin{array}{l}\text { 10. Un jour sa mère } \\
\text { ayant fait et cuit des } \\
\text { galettes, lui dit: }\end{array}$ & $\begin{array}{l}\text { 10. Un día, su madre le } \\
\text { había cocido y hecho } \\
\text { le dijo: } \\
\text { * ¿gallette? -crepa } \\
\text { salada, pastelillo, } \\
\text { galleta. }\end{array}$ & $\begin{array}{l}\text { 10. Un día, su mamá, } \\
\text { que había hecho } \\
\text { pastelillos le dijo: }\end{array}$ \\
\hline & $\begin{array}{l}\text { 11. Va voir comme se } \\
\text { porte ta mère-grand, }\end{array}$ & $\begin{array}{l}\text { 11. - Ve a ver cómo está } \\
\text { tu abuela, }\end{array}$ & $\begin{array}{l}\text { 11. - Ve a ver cómo está } \\
\text { tu abuelita, }\end{array}$ \\
\hline & $\begin{array}{l}\text { 12. car on m'as dit qu'elle } \\
\text { était malade, }\end{array}$ & $\begin{array}{l}\text { 12. pues me han dicho que } \\
\text { estaba enferma. }\end{array}$ & $\begin{array}{l}\text { 12. pues me han dicho que } \\
\text { estaba enferma. }\end{array}$ \\
\hline & $\begin{array}{l}\text { 13. porte-lui une galette et } \\
\text { ce petit pot de beurre. }\end{array}$ & $\begin{array}{l}\text { 13. Lévale un } \\
\text { y este tarro de } \\
\text { mantequilla. }\end{array}$ & $\begin{array}{l}\text { 13. Llévale un pastelillo } \\
\text { y este tarro de } \\
\text { mantequilla. }\end{array}$ \\
\hline
\end{tabular}




\begin{tabular}{|c|c|c|c|}
\hline & $\begin{array}{l}\text { 14. Le Petit Chaperon } \\
\text { rouge partit aussitôt } \\
\text { pour aller chez sa } \\
\text { mère-grand, }\end{array}$ & $\begin{array}{l}\text { 14. Caperucita partió } \\
\text { inmediatamente para ir } \\
\text { a casa de su abuela, }\end{array}$ & $\begin{array}{l}\text { 14. Caperucita Roja partió } \\
\text { enseguida hacia la } \\
\text { casa de su abuelita, }\end{array}$ \\
\hline & $\begin{array}{l}\text { 15. qui demeurait dans un } \\
\text { autre village. }\end{array}$ & $\begin{array}{l}\text { 15. quien moraba en otra } \\
\text { aldea. }\end{array}$ & 15. que vivía en otra aldea \\
\hline \multirow[t]{13}{*}{ Isis } & $\begin{array}{l}\text { 1. Le Petit Chaperon } \\
\text { rouge. } \\
\text { Charles Perrault }\end{array}$ & $\begin{array}{l}\text { 1. Caperucita Roja. } \\
\text { Charles Perrault }\end{array}$ & $\begin{array}{l}\text { 1. Caperucita Roja. } \\
\text { Charles Perrault }\end{array}$ \\
\hline & 2. Il était une fois & 2. Erase una vez & 2. Érase una vez \\
\hline & $\begin{array}{l}\text { 3. une petite fille de } \\
\text { Village, }\end{array}$ & $\begin{array}{l}\text { 3. una pequeña niña de } \\
\text { campo, }\end{array}$ & $\begin{array}{l}\text { 3. una pequeña niña de } \\
\text { campo, }\end{array}$ \\
\hline & $\begin{array}{l}\text { 4. la plus jolie qu' on eût } \\
\text { su voir; }\end{array}$ & $\begin{array}{l}\text { 4. la más bella (que } \\
\text { nuestros ojos } \\
\text { pudieran ver) de la } \\
\text { región; }\end{array}$ & $\begin{array}{l}\text { 4. la más bella que se } \\
\text { pudiera ver. }\end{array}$ \\
\hline & $\begin{array}{l}\text { 5. sa mère en était folle, } \\
\text { et sa mère-grand plus } \\
\text { folle encore. }\end{array}$ & $\begin{array}{l}\text { 5. si su mamá estaba } \\
\text { loca, su abuela estaba } \\
\text { aún más. }\end{array}$ & $\begin{array}{l}\text { 5. Su mamá la amaba } \\
\text { con locura y su } \\
\text { abuelita aún más. }\end{array}$ \\
\hline & $\begin{array}{l}\text { 6. Cette bonne femme } \\
\text { lui fit faire un petit } \\
\text { chaperon rouge, }\end{array}$ & $\begin{array}{l}\text { 6. La abuela bondadosa } \\
\text { le hizo una pequeña } \\
\text { capucha }\end{array}$ & $\begin{array}{l}\text { 6. La dulce abuelita } \\
\text { le confeccionó una } \\
\text { pequeña caperuza roja }\end{array}$ \\
\hline & 7. qui lui seyait si bien, & $\begin{array}{l}\text { 7. que le sentaba muy } \\
\text { bien }\end{array}$ & $\begin{array}{l}\text { 7. que le sentaba muy } \\
\text { bien }\end{array}$ \\
\hline & 8. que partout & 8. y en todos lados & 8. y por todos lados \\
\hline & $\begin{array}{l}\text { 9. on l'appelait le Petit } \\
\text { Chaperon rouge. }\end{array}$ & $\begin{array}{l}\text { 9. se le conoció como } \\
\text { "Caperucita roja". }\end{array}$ & $\begin{array}{l}\text { 9. le llamaban Caperucita } \\
\text { Roja. }\end{array}$ \\
\hline & $\begin{array}{l}\text { 10. Un jour sa mère } \\
\text { ayant fait et cuit des } \\
\text { galettes, lui dit: }\end{array}$ & $\begin{array}{l}\text { 10. Un día la mamá de } \\
\text { Caperucita roja le hizo } \\
\text { unas crepas saladas y } \\
\text { le dijo: }\end{array}$ & $\begin{array}{l}\text { 10. Un día la mamá de } \\
\text { Caperucita le preparó } \\
\text { unos pastelitos y le } \\
\text { dijo: }\end{array}$ \\
\hline & $\begin{array}{l}\text { 11. Va voir comme se } \\
\text { porte ta mère-grand, }\end{array}$ & $\begin{array}{l}\text { 11. Ve a ver como sigue tu } \\
\text { abuela, }\end{array}$ & $\begin{array}{l}\text { 11. Ve a ver cómo está tu } \\
\text { abuelita, }\end{array}$ \\
\hline & $\begin{array}{l}\text { 12. car on m'as dit qu'elle } \\
\text { était malade, }\end{array}$ & $\begin{array}{l}\text { 12. pues me dijo que es- } \\
\text { taba enferma, llévale } \\
\text { una crepa }\end{array}$ & $\begin{array}{l}\text { 12. pues me dijeron que } \\
\text { estába enferma. }\end{array}$ \\
\hline & $\begin{array}{l}\text { 13. porte lui une galette et } \\
\text { ce petit pot de beurre. }\end{array}$ & $\begin{array}{l}\text { 13. y este pequeño tarro de } \\
\text { mantequilla. }\end{array}$ & $\begin{array}{l}\text { 13. Llévale este pastelito } \\
\text { y este pequeño tarro } \\
\text { con mantequilla. }\end{array}$ \\
\hline
\end{tabular}




\begin{tabular}{|c|c|c|c|}
\hline $\begin{array}{c}\text { 14. Le Petit Chaperon } \\
\text { rouge partit aussitôt } \\
\text { pour aller chez sa } \\
\text { mère-grand, }\end{array}$ & $\begin{array}{c}\text { 14. Caperucita roja partió } \\
\text { lo más pronto posible } \\
\text { a la casa de su abuela, }\end{array}$ & $\begin{array}{c}\text { 14. Caperucita Roja partió } \\
\text { lo más pronto posible } \\
\text { a la casa de su abuelita }\end{array}$ \\
\cline { 2 - 4 } & $\begin{array}{c}\text { 15. qui demeurait dans un } \\
\text { autre village. }\end{array}$ & $\begin{array}{l}\text { 15. quien vivía en otra } \\
\text { aldea. }\end{array}$ & $\begin{array}{c}\text { 15. quien vivía en otra } \\
\text { aldea. }\end{array}$ \\
\hline
\end{tabular}

\section{Cohesión verbal}

Entre los retos que encierra este primer párrafo, encontramos los tiempos verbales. En francés, la perífrasis verbal de la oración la plus jolie qu'on ê̂t su voir, es un plus que parfait de subjuntivo, mientras que en español es un pretérito imperfecto de subjuntivo. Además, encontramos la perífrasis verbal en la oración cette bonne femme lui fit faire, donde fit es un imparfait de subjuntivo más el infinitivo faire, ambos del verbo hacer. También se encuentra la forma seyait, del verbo seoir, el cual ya no se utiliza actualmente, con excepción, quizá, para la tercera persona del presente del condicional y del subjuntivo, y en el participio presente.

Para eût su voir, en su mayoría los estudiantes optaron por traducirlo como «que se haya visto», el cual es un pretérito perfecto compuesto del modo subjuntivo; una estudiante, por otro lado, optó por «que se hubiera visto», el cual es un pretérito pluscuamperfecto del subjuntivo, y finalmente, Aris e Isis hicieron una traducción más libre y tradujeron «de la cual se haya tenido noticia» y «que nuestros ojos pudieran ver», respectivamente. Lo anterior en la primera versión de su traducción. En la última versión la mayoría conservó el «haya visto» o «hubiera visto» y ambas traducciones se consideraron equivalencias apropiadas del francés.

En el caso de fit faire, casi todos los estudiantes incurrieron en un error al traducir «le tejió» o «le hizo»; sólo una estudiante tradujo correctamente como «le mandó a hacer». La frase fit faire en francés indica que el sujeto no hace la acción, sino que pide a alguien que la realice. Este es un importante error de comprensión del texto que da lugar a confusión y cambia el sentido de la historia.

Para qui lui seyait si bien cuatro estudiantes eligieron traducir seyait por «quedaba» y dos por «sentaba». Es importante notar que, a pesar de ser un tiempo verbal en desuso, los estudiantes lo tradujeron correctamente, pues el verbo seoir significa «favorecer»o «sentar bien».

Las perífrasis verbales pueden representar un reto para el traductor, ya que constituyen tiempos verbales complejos. Sin embargo, podemos notar que en primera instancia los estudiantes se acercaron a ellas de manera correcta y gracias a la corrección en clase y la interacción con sus compañeros llegaron a una traducción final aceptable. 


\section{Semántica}

Uno de los problemas de semántica más graves, fue la traducción del enunciado de sa mère en était folle, et sa mère-grand plus folle encore. Cuatro estudiantes incurren en un contrasentido grave al traducir como: «su madre estaba enferma y su abuela todavía más» o «si su mamá estaba loca, su abuela lo estaba aún más», porque olvidan la función de en. En la oración, en sustituye a une petite fille. No obstante, al final llegan a una traducción correcta: «su madre estaba loca por ella y su abuela aún más», o bien, «su madre la amaba con locura y su abuela todavía más».

Durrenmat (2012: 2) señala que en las oraciones sa mère en était folle et sa mère-grand plus folle encore, hay que identificar en primer lugar la naturaleza de en, llamado pronombre adverbial debido a su origen etimológico y su invariabilidad, la cual pertenece a la clase de los pronombres personales y se coloca siempre antes del verbo. En segundo lugar — añade_-, aquí se trata de una anáfora (figura retórica que consiste en repetir una palabra o conjunto de palabras al inicio de una frase o verso), puesto que en toma sentido apoyándose en el grupo nominal une petite fille de village, donde el pronombre funge como expansión del adjetivo «loca»: «su mamá estaba loca por esa niña».

Sin embargo, la norma gramatical contemporánea prohíbe el uso del pronombre en cuando se habla de un ser humano e impone que se utilice la preposición de seguida de un pronombre personal: «de ella». Esta regla no aparece en el francés clásico, el cual era mucho más libre en lo que se refiere al empleo de términos pronominales. También podría ser un error de contexto cultural y para corregirlo fue indispensable la intervención de la profesora. Por lo tanto, representa un excelente ejemplo de la importancia de la interacción profesor-estudiante.

\section{Lexicología}

El léxico en Le Petit Chaperon Rouge refleja el uso de la lengua francesa, en diferentes épocas los editores intervinieron para que desaparecieran algunas palabras o giros de lenguaje consideradas obsoletas o incomprensibles. Fue así como: « $S a$ mère ayant cuit et fait des galettes » se convirtió poco a poco en: «Sa mère ayant fait et cuit des galettes » o simplemente en: «Sa mère ayant fait des galettes » (Durrenmat, 2012).

En la traducción del cuento, todos los estudiantes tuvieron problemas con la palabra galette. La tradujeron como: «tartas», «galletas», «panquecillos», «panquecitos», «pastelitos», «pastelillos», «crepas» o bien dejaron una línea en blanco ante su imposibilidad para traducir esa palabra.

Los diccionarios en línea como WordReference señalan que galette es «arepa», «enchilada», «guita», «roscón», «taco» o «torta». En el diccionario Larousse francés- 
español aparece «torta, crepa salada, roscón de Reyes o pasta». De la misma manera se encontraron resultados semejantes en diversos diccionarios, por lo que algunos estudiantes optaron por traducir de acuerdo con la semejanza de la palabra en español: galette $=\langle$ galleta $\rangle$.

En el sitio del Centre National de Ressources Textuelles et Lexicales (Centro Nacional de Recursos Textuales y Lexicales) ${ }^{1}$ dependiente del Centre National de la Recherche Scientifique en Francia, encontramos: «es un pastel redondo elaborado con pasta hojaldrada y cocido al horno, al principio era un pastel casero que se transforma a través del tiempo en un ideal de elegancia burguesa; antes de la Revolución se salía a comer 'galette, beber vino y bailar'».

Más adelante nos dimos a la tarea de ver cómo había sido traducido este término en los cuentos publicados y encontramos: «tortas», «pasteles» o «galletas». Tras realizar esta investigación, finalmente los estudiantes optaron por usar «pastelillo» o «pastelitos» en sus traducciones finales.

Por otro lado, en su primera versión dos estudiantes traducen beurre como «mermelada», lo cual es una falta grave, ya que beurre significa mantequilla. Asimismo traducen petit pot por «bote», mientras que otros optan por «frasco», «frasquito» o «tarro». Estos errores se corrigieron en la versión final.

Como podemos ver, el análisis nos brinda elementos muy ricos para observar el proceso de traducción de los estudiantes. En este caso, apenas hemos cubierto los errores más importantes en los que incurrieron en este primer párrafo. Para ejemplificar como podemos llegar a un análisis aún más profundo, a continuación demostraremos cómo observamos su proceso de traducción gracias a la herramienta informática El Espía.

\subsection{ANÁLISIS DE LA TRADUCCIÓN DE UN ESTUDIANTE CON EL ESPÍA}

Ya hemos mencionado que El Espía representó una gran ventaja para observar el proceso cognitivo y psicolingüístico de la traducción de los estudiantes. Esta herramienta funciona de la siguiente manera: los estudiantes utilizaron este programa en sus computadoras portátiles para llevar a cabo la tercera, cuarta versión y hasta quinta versión de la traducción del cuento. Una vez que terminaron, la profesora pudo observar en su propia computadora cuál fue el proceso exacto que siguió cada estudiante. El Espía proporciona dos elementos importantes: 1) el reporte detallado, segundo a segundo, de todos los comandos que utilizó el estudiante (agregar, suprimir, borrar, insertar, etc.) y 2) una reconstrucción a modo de video de las acciones exactas que realizó el estudiante ${ }^{2}$.

\footnotetext{
1 http://www.cnrtl.fr/portail/

2 En el siguiente enlace se puede leer la información completa acerca de El Espía e incluso descargar el programa para observar su funcionamiento: http://www.uv.mx/blogs/elespia/author/ albramirez/
} 
A continuación presentaremos un ejemplo de dicho reporte y cómo nos ayuda a conocer el proceso de traducción del estudiante.

\begin{tabular}{|c|c|l|l|}
\hline \multicolumn{4}{|c|}{ Reporte } \\
\hline Tiempo & Ubicación & \multicolumn{1}{|c|}{ Operación } & \multicolumn{1}{c|}{ Cadena } \\
\hline 00:00:00-00:00:00 & $0-0$ & Agregar & E \\
\hline 00:00:00-00:00:00 & & Pausa de 00:00:07 & \\
\hline 00:00:07-00:00:16 & $1-27$ & Agregar & rase una vez una niñita de \\
\hline 00:00:17-00:00:18 & $27-25$ & Eliminar & Ed \\
\hline 00:00:18-00:00:22 & $25-47$ & Agregar & que vivía en una aldea \\
\hline 00:00:23-00:00:23 & $47-47$ & Eliminar & \\
\hline 00:00:24-00:00:27 & $47-55$ & Agregar & la nini \\
\hline 00:00:28-00:00:28 & $55-54$ & Eliminar & In \\
\hline 00:00:29-00:00:36 & $54-89$ & Agregar & ñita más bella que se hubiera visto \\
\hline
\end{tabular}

El cuadro anterior sólo demuestra la primera oración del cuento. El reporte es extenso y cubre cada segundo del proceso que siguió Daniel para traducir el primer párrafo. Sin embargo, se puede identificar toda la riqueza de información que nos brinda esta herramienta.

En la reconstrucción observamos que luego de introducir la E mayúscula con que inicia el texto, hay una pausa de siete segundos durante los cuales Daniel duda, tal vez, si traducir el inicio como «Había una vez», tal como estaba en su primera versión, o si elegir la opción que finalmente adopta: «Érase una vez». Luego piensa en traducir «una niñita de aldea» y lo cambia por «una niñita que vivía en una aldea».

Por otra parte, hay un detalle muy interesante que no se aprecia bien en el reporte: cuando Daniel escribe el contrasentido «la mama de Caperucita estaba loca», lo cambia de inmediato. De la misma forma, escribe, sin darse cuenta del error, el acento de «mamá» en la primera «a», lo cual se atribuye a que quizá haya una interferencia con la palabra mère en francés, la cual lleva el acento en la primera vocal.

También duda cuando traduce «esta buena mujer le hizo» y opta por «esta buena mujer le tejió», la cual es una traducción errónea, ya que en ningún momento aparece la palabra tisser en francés (que significa «tejer» en español) y tampoco en el original se indica que la abuela le «hizo» o le «tejió», sino que «le mandó a hacer».

La oración Un jour sa mère ayant cuit et fait des galettes la traduce, en la primera versión como «Un día su madre habiendo cocinado panquecillos», mientras en la versión con El Espía opta por: «Un día que su madre había hecho pastelillos». El cambio puede deberse a que en uno de los seminarios de traducción se leyó un artículo sobre los usos correctos e incorrectos del gerundio y quizá esto hizo reflexionar al estudiante. 
Luego Daniel continúa con la siguiente oración: Va voir comme se porte ta mèregrand, car on m'a dit qu'elle était malade que traduce: "Ve a ver cómo se encuentra tu abuela, pues me han dicho que se encuentra enferma» y en la versión final escribe: «ve a ver como está tu abuela, porque me dijeron que estaba enferma». A pesar de que olvida el acento en la palabra «cómo», se observa que su traducción es mejor que en su versión anterior.

Así podemos afirmar que la herramienta El Espía puede contribuir a realizar un análisis a profundidad del proceso que siguen los estudiantes al traducir un texto. Esto es fundamental para realizar un análisis microgenético porque vemos cada detalle del proceso cognitivo del estudiante. Podemos aprender mucho más de las pausas, los errores y las omisiones en las que incurre el estudiante que del texto final en sí, donde sólo vemos sus errores finales.

\section{CONCLUSIONES PRELIMINARES}

El análisis microgenético permite ver los cambios en el aprendizaje de los estudiantes durante un tiempo corto y en una situación particular como se explicó anteriormente.

Mediante el análisis de las distintas versiones de la traducción de los estudiantes en combinación con la herramienta informática El Espía, se pudo observar cada detalle del proceso que siguen para traducir y los cambios que se producen en su aprendizaje.

Los resultados encontrados mediante el análisis microgenético permitieron comprobar progresos importantes en el aprendizaje de la traducción.

La versión final del texto de cada estudiante denota una mejora de su primera versión y se acerca a lo que podríamos considerar una traducción aceptable.

La interacción profesora-estudiante y estudiante-estudiante resultó muy favorable ya que a medida que fueron avanzando en la traducción, dejó de ser necesario elaborar cuatro o cinco borradores de cada párrafo porque su traducción era correcta al segundo o tercer intento y así pudimos darnos cuenta de que habían aprovechado el andamiaje proporcionado en la interacción.

En síntesis, los resultados obtenidos demuestran que la interacción con el adulto experimentado permite facilitar el proceso de aprendizaje de la traducción y al mismo tiempo se pudo comprobar que la evaluación que se iba realizando de manera individualizada, sesión por sesión, ofrecía información relevante sobre las diferencias en el proceso de aprendizaje.

Por lo tanto, el análisis microgenético con el apoyo de El Espía contribuye a descubrir el proceso de aprendizaje de la traducción. 


\section{BIBLIOGRAFÍA}

BALSLEV, Kristine (2006): Microgenèses didactiques dans une situation de révision textuelle en milieu adulte, (Thèse du doctorat No. 370). Ginebra: Faculté de psychologie et des sciences de l'éducation, Université de Genève.

BAlsLev, Kristine; SAADA-RoBerT, Madelon (2006): «Les microgenèses situées. Unités et procédés d'analyse inductive-déductive ». Recherches qualitatives, 26/2: 85-109.

BERMEJo, Vicente (2005): «Microgénesis y cambio cognitivo: adquisición del cardinal numérico». Psicotema, 17/4. URL: redalyc.uaemex.mx/src/inicio/ArtPdfRed.jsp?iCve $=72717403[9 / 07 /$ 2011].

DurRenMAT, Jacques (2012): Explication de texte : La Question de Grammaire. URL: http://trf.education.gouv.fr/pub/edutel/siac/siac2/jury/2004/capes_ext/lettres/et2.pdf [03/02/2012].

Nord, Christiane (1998): « La unidad de traducción en el enfoque funcionalista ». Quaderns, 1.

Perrault, Charles (2011): Le Petit Chaperon rouge. URL: chaperonrouge online.fr/perraultfr.htm [30/06/2012].

Ramírez, Alberto; VACA, Jorge (2011): «El Espía 2.0: Herramienta digital para la exploración de los procesos de producción de textos ». In: Memoria del XI Congreso Nacional de Investigación Educativa. México: COMIE.

Rosenthal, Victor (2011): «Approche microgénétique du langage et de la perception ». Texto. URL: http://www.revue-texto.net/Inedits/Rosenthal/Rosenthal_Micro1.html. [11/12/2011].

Siegler, Robert; Crowley, Kevin (1992): «Microgenetic methods revisited». Psychologist, 47/10: 1241-43.

Vigotsky, Lev (1978): Pensamiento y lenguaje. Madrid: Paidós. 